\title{
Visual Impairment, Eye Diseases, and Dementia Risk: A Systematic Review and Meta-Analysis
}

Handling Associate Editor: Ruth Peters

\author{
Elżbieta Kuźma ${ }^{\mathrm{a}, 1, *}$, Thomas J. Littlejohns ${ }^{\mathrm{b}, 1}$, Anthony P. Khawaja ${ }^{\mathrm{c}}$, David J. Llewellyn ${ }^{\mathrm{d}, \mathrm{e}}$, \\ Obioha C. Ukoumunne ${ }^{\mathrm{f}}$ and Ulrich Thiem ${ }^{\mathrm{a}, \mathrm{g}}$ \\ ${ }^{a}$ Albertinen-Haus Centre for Geriatrics and Gerontology, University of Hamburg, Hamburg, Germany \\ ${ }^{\mathrm{b}}$ Nuffield Department of Population Health, University of Oxford, Oxford, United Kingdom \\ ${ }^{\mathrm{c}}$ NIHR Biomedical Research Centre at Moorfields Eye Hospital NHS Foundation Trust \& UCL Institute of \\ Ophthalmology, London, United Kingdom \\ ${ }^{\mathrm{d}}$ College of Medicine and Health, University of Exeter, Exeter, United Kingdom \\ ${ }^{\mathrm{e}}$ Alan Turing Institute, London, United Kingdom \\ ${ }^{\mathrm{f}}$ NIHR ARC South West Peninsula (PenARC), University of Exeter Medical School, Exeter, United Kingdom \\ ${ }^{\mathrm{g}}$ University Medical Center Hamburg-Eppendorf, Hamburg, Germany
}

Accepted 7 July 2021

\begin{abstract}
.
Background: Visual impairment and eye diseases have been associated with dementia, though with mixed findings and often in cross-sectional studies.

Objective: To identify prospective studies investigating associations between visual impairment or common eye diseases and risk of all-cause dementia or key dementia subtypes.

Methods: We searched Medline, PsycINFO, and Embase from inception to January 2020. We also conducted backward and forward citation searches of included studies and set up alerts to identify studies published after the search date. Random-effects meta-analysis was used to combine adjusted estimates across studies.

Results: Thirty studies met our eligibility criteria. For visual impairment, pooled estimates indicated an increased risk of allcause dementia $\left(37,705\right.$ participants, 3,415 cases, risk ratio $[\mathrm{RR}]=1.38,95 \%$ confidence interval $\left.[\mathrm{CI}]: 1.19-1.59, \mathrm{I}^{2}=28.6 \%\right)$. Pooled estimates also suggested an increased dementia risk associated with cataract (6,659 participants, 1,312 cases, hazard ratio $\left.[\mathrm{HR}]=1.17,95 \% \mathrm{CI} 1.00-1.38, \mathrm{I}^{2}=0.0 \%\right)$ and diabetic retinopathy $(43,658$ participants, 7,060 cases, $\mathrm{HR}=1.34,95 \%$ CI 1.11-1.61, $\left.\mathrm{I}^{2}=63.9 \%\right)$, respectively. There was no evidence of an association between glaucoma (175,357 participants, 44,144 cases, $\left.\mathrm{HR}=0.97,95 \% \mathrm{CI} 0.90-1.04, \mathrm{I}^{2}=51.5 \%\right)$ or age-related macular degeneration $(7,800,692$ participants, $>2,559$ cases, $\mathrm{HR}=1.15,95 \% \mathrm{CI} 0.88-1.50, \mathrm{I}^{2}=91.0 \%$ ) and risk of dementia, respectively.

Conclusion: As visual impairment, cataract, and diabetic retinopathy are associated with an increased likelihood of developing dementia, early diagnosis may help identify those at risk of dementia. Given most causes of visual impairment are treatable or preventable, the potential for dementia prevention warrants further investigation.
\end{abstract}

Keywords: Alzheimer's disease, dementia, eye diseases, prospective studies, vision disorders

\footnotetext{
${ }^{1}$ These authors contributed equally to this work.

*Correspondence to: Dr Elżbieta Kuźma, Albertinen-Haus Centre for Geriatrics and Gerontology, Sellhopsweg 18-22,
}

22459 Hamburg, Germany. Tel.: +49 405581 4932; E-mail: elzbieta.kuzma@immanuelalbertinen.de. 


\section{INTRODUCTION}

Identifying modifiable risk factors for dementia is of great importance given the lack of cure, with recent evidence recognizing sensory impairment including hearing and visual impairment (VI) as promising targets $[1,2]$. Hearing loss has been highlighted as a key modifiable dementia risk factor by both Lancet Commissions $[3,4]$ on dementia prevention, intervention, and care. The evidence on VI is less well studied. VI is most frequently caused by uncorrected refractive errors and eye diseases (e.g., cataract, glaucoma), and typically defined by visual acuity [5]. VI is common at older ages, with 20-22\% prevalence in those aged 70 and over [6]; however, in contrast to dementia, there are effective interventions for VI, with $80 \%$ of its causes being treatable (e.g., corrective lenses) or curable (e.g., cataract surgery) [5].

A recent meta-analysis of data from 14 and 12 prospective studies, respectively found visual impairment including color vision deficiency was associated with an increased risk of dementia (Risk Ratio $[\mathrm{RR}]=1.47,95 \%$ Confidence Interval $[\mathrm{CI}]$ : 1.36-1.60) and cognitive impairment $(\mathrm{RR}=1.35$, 95\% CI: 1.28-1.41) [7]. A further meta-analysis of seven observational studies found glaucoma was associated with an increased risk of Alzheimer's disease (AD) $(\mathrm{RR}=1.24,95 \% \mathrm{CI}: 1.02-1.51)$ [8]. However, restricting to five prospective studies resulted in no association between glaucoma and AD [8]. Similarly, a recent meta-analysis of one case-control and one cross-sectional study suggested an association between age-related macular degeneration (AMD) and cognitive impairment (Odds Ratio $[\mathrm{OR}]=2.42$, 95\% CI: $1.06-5.56$ ), whereas pooled results of two prospective studies indicated no association with $\mathrm{AD}(\mathrm{RR}=1.27,95 \% \mathrm{CI}$ : 0.53-3.04) [9]. Another systematic review found retinal microvascular changes were associated with dementia, cognitive dysfunction, and neuroimaging abnormalities, though most included studies were cross-sectional and no meta-analysis was conducted [10].

Recently, various prospective studies have been conducted which may help to clarify whether VI based on visual acuity is an independent dementia risk factor. Therefore, we conducted the first systematic review and meta-analysis of prospective studies investigating VI and common eye diseases, which cause VI, in relation to incident all-cause dementia or key dementia subtypes.

\section{METHODS}

Our systematic review was conducted according to the guidance provided by the Centre for Reviews and Dissemination (CRD, UK) [11]. Where possible, we performed random effects meta-analyses.

Search strategy, study selection, and data extraction

Following a pre-defined protocol (Supplementary Methods) and based on previous systematic reviews $[6,12,13]$, we developed search strategies for Medline, PsycINFO, and Embase (via OvidSP) including subject headings and free text terms relevant to VI, common eye diseases, dementia, key dementia subtypes $(\mathrm{AD}$, vascular dementia $(\mathrm{VaD})$, dementia with Lewy bodies, frontotemporal dementia) and study design (Supplementary Figures 1-3). We conducted searches on 8 January 2020 (EK) supplemented by forward and backward citation searches of included publications and ongoing alerts to identify studies published after the search date. We included prospective studies in adults published in English investigating the association between VI or eye diseases and incident all-cause dementia or key dementia subtypes. The comparison group was no VI or in studies of eye disease, the eye disease of interest was not in the comparison group. We excluded studies with outcomes other than dementia or key dementia subtypes, studies using only a single cognitive measure or only self-reported dementia to define the outcome, studies investigating aspects of visual perception (e.g., color vision deficiency), studies with no comparison group or a comparison group other than no VI or no eye disease, and studies that were not prospective (e.g., case-control, cross-sectional, or retrospective studies). Animal studies, case reports, narrative reviews, letters, editorials, opinions, book chapters, conference abstracts, non-peer-reviewed publications, and duplicate publications using the same data were excluded. Two reviewers (EK, TJL) independently screened titles and abstracts, and reviewed full texts based on pre-defined inclusion and exclusion criteria discussing any discrepancies with a third reviewer (UT).

Study characteristics and fully adjusted results were extracted by one reviewer (EK) and checked by the second (TJL). Corresponding authors of 26 studies were contacted for clarification or additional data, with 11 providing a response (details in Supplementary Methods). Risk of bias of included studies 
was assessed independently by the same two reviewers (EK, TJL) using the Quality Assessment Tool for Quantitative Studies [14] with discrepancies resolved by discussion. Overall risk of bias and potential sources of bias (selection bias, study design, confounders, blinding, data collection, withdrawals, and drop-outs [attrition bias]) were rated as strong, moderate, or weak according to the dictionary provided with the tool [14]. Detailed description of component ratings is provided in Supplementary Methods. In accordance with the tool, studies with no weak component ratings received a strong overall rating. Moderate and weak overall ratings were assigned to studies with only one and two or more weak component ratings, respectively.

\section{Data analysis}

Included studies were categorized based on exposure into those examining VI and eye diseases and outcome into all-cause dementia and key dementia subtypes. VI assessed with visual acuity was categorized into: no VI $(\leq 0.3$ Logarithm of the Minimum Angle of Resolution [LogMAR]), mild VI (0.3 $<\operatorname{LogMAR} \leq 0.5)$, moderate VI $(0.5<\operatorname{LogMAR}$ $\leq 1.0)$, severe VI $(1.0<\operatorname{LogMAR} \leq 1.3)$, or blindness $(1.3<\operatorname{LogMAR})$ based on the World Health Organization (WHO) classification [5]. Studies where the comparison group was categorized as "no VI' and the exposure as 'mild VI' or 'mild VI and worse' based on visual acuity assessment or self-report were added to a meta-analysis investigating the association between VI and dementia risk. We also conducted meta-analyses of the association between each eye disease and dementia risk. Given the heterogeneity between studies, we performed random effects meta-analyses using the metan command [15] in Stata 15.1 (StataCorp, College Station, TX, USA). Heterogeneity was investigated using Cochran's Chi-squared test and the I-squared statistic [16]. If multiple studies investigated the same exposure and outcome using overlapping data sources, we prioritized studies with a higher overall quality rating, bigger sample size, and published more recently. Pooled estimates are presented as hazard ratios (HRs) if studies included in meta-analysis reported only HRs. If included studies reported a mixture of HRs, RRs, and standardized rate ratios, we present pooled estimates as RRs [17]. Studies that could not be included in meta-analyses due to important methodological differences were synthesized narratively. In sensitivity analyses, we re-examined associations according to exposure ascertainment by repeating the VI and all-cause dementia meta-analysis after excluding studies with self-reported VI and pooling results for studies with low contrast sensitivity, open-angle glaucoma, and primary open-angle glaucoma as the exposures, respectively. We also repeated the main meta-analyses after excluding studies with a global risk of bias rating of weak if at least two studies with a global rating of moderate or strong remained.

\section{RESULTS}

Our searches resulted in 4,185 records. After removing 1,034 duplicates, 3,104 records were removed based on title and abstract screening resulting in 47 publications for full-text review, of which 21 met our inclusion criteria. Nine additional studies were identified via backward citation searches and alerts (Fig. 1) totaling in 30 included studies.

Key characteristics of included studies are provided in Table 1. Nine studies were from the United States, eight from Europe, and thirteen from Asia. Seventeen studies used electronic medical record datasets, eleven used data from population-based cohorts, and two performed post-hoc observational analyses of randomized controlled trial cohorts. Twenty-four studies used medical records $(n=16)$ or ophthalmic examination $(n=8)$ to identify VI or eye diseases, whereas six studies used self-report only $(n=3)$ or a combination of self-report and ophthalmic examination $(n=2)$, or a combination of self-report and medical records $(n=1)$. All-cause dementia or key dementia subtypes were ascertained based on medical records or a combination of sources (Supplementary Tables 2-7). Analytic sample sizes varied between the studies from 812 in a cohort study [18] to $7,766,857$ in the biggest medical records study [19].

\section{Risk of bias}

Overall, three studies were rated as strong quality, 11 as moderate and 16 as weak (Supplementary Table 1). Studies with a moderate rating did not describe the validity and reliability of data collection method $(n=7)$ or were subject to potential attrition $(n=3)$ or selection bias $(n=1)$. Studies with a weak rating were subject to a combination of potential sources of bias including confounding $(n=11)$, validity and reliability of data collection method $(n=10)$, attrition $(n=9)$, selection bias $(n=6)$, and blinding $(n=1)$. 


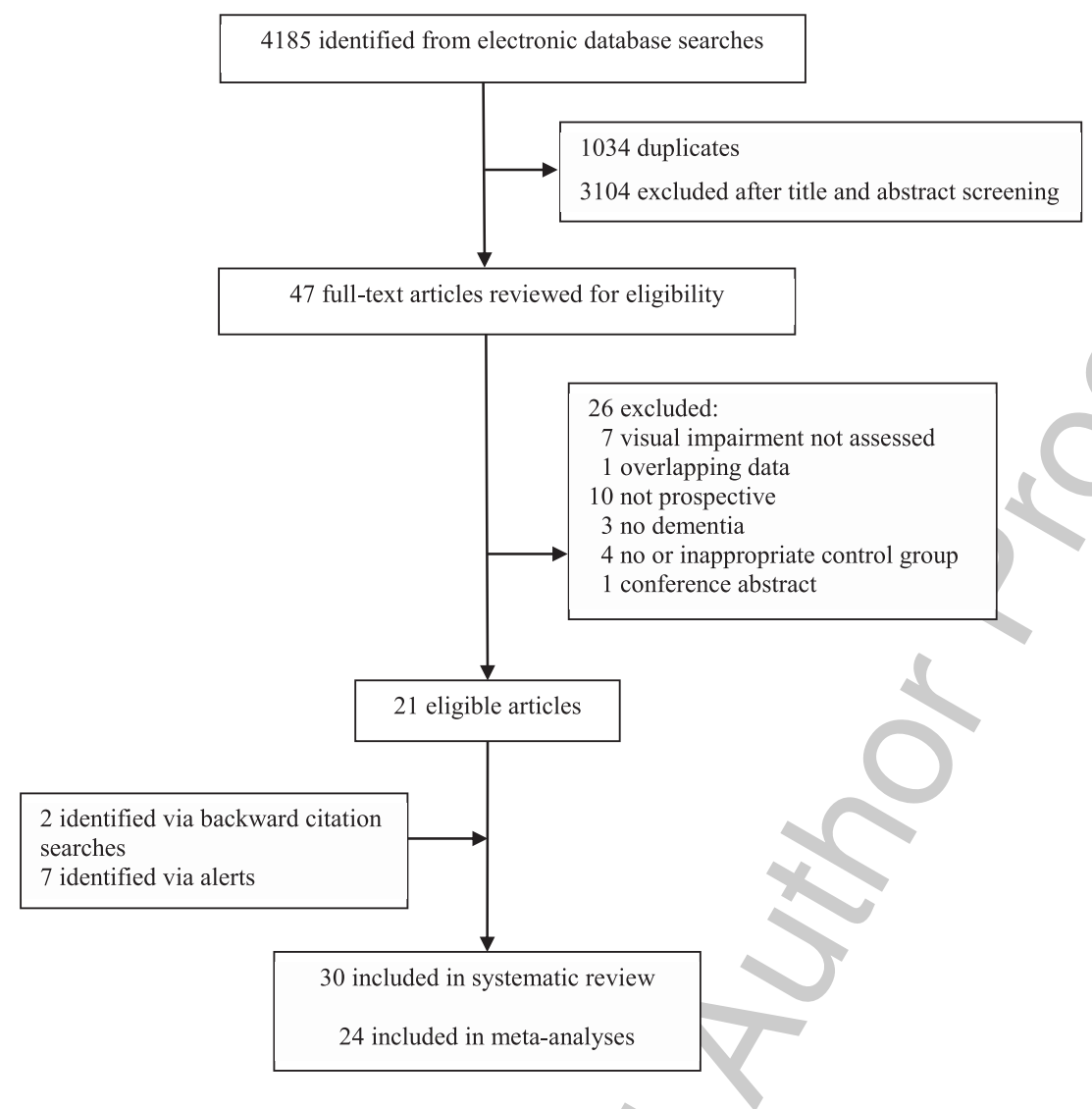

Fig. 1. Flowchart of search results and study retrieval.

\section{Visual impairment and eye diseases}

A narrative synthesis of additional findings on visual impairment, glaucoma, AMD, and diabetic retinopathy that could not be included in metaanalyses is provided in Supplementary Results.

\section{Visual impairment}

Ten cohort studies investigated the association between VI and incident all-cause dementia [20-29] (Supplementary Table 2). The pooled estimate of seven studies [20, 21, 23, 25-27, 29] indicated a higher risk of all-cause dementia in those with at least mild VI compared to no VI (37,705 participants, 3,415 dementia cases, pooled adjusted $\mathrm{RR}=1.38$, 95\% CI: $1.19-1.59, p<0.001, \mathrm{I}^{2}=28.6 \%$; Fig. 2 ). After excluding three studies [21, 26, 29] using selfreported VI, the pooled estimate remained similar (26,381 participants, 2,651 dementia cases, pooled adjusted $\mathrm{HR}=1.35$, 95\% CI: $1.10-1.67, p=0.005$,
$\mathrm{I}^{2}=38.7 \%$; Supplementary Figure 4$)$. The pooled estimate of two studies [20,22] that assessed VI based on contrast sensitivity was also similar to our main finding although not statistically significant $(3,892$ participants, 565 dementia cases, pooled adjusted $\mathrm{HR}=1.42$, 95\% CI: $0.82-2.48, p=0.21, \mathrm{I}^{2}=79.7 \%$; Supplementary Figure 5).

Three studies [28-30] investigated the association with incident AD (Supplementary Table 2). The pooled estimate of two studies [28, 29] indicated an increased risk of $\mathrm{AD}$ in those with at least mild VI compared to no VI $(6,031,708$ participants, 123,717 AD cases, pooled adjusted $\mathrm{HR}=1.47,95 \%$ CI: $1.43-1.50, p<0.001, \mathrm{I}^{2}=0.0 \%$; Supplementary Figure 6). Two studies [28, 29] investigated the association with $\mathrm{VaD}$ (Supplementary Table 2). Similarly, the pooled estimate of these two studies $[28,29]$ indicated an increased risk of $\mathrm{VaD}$ in those with at least mild VI compared to no VI $(6,031,708$ participants, 20,764 VaD cases, pooled adjusted $\mathrm{HR}=1.40,95 \%$ CI: $1.33-1.47, p<0.001, \mathrm{I}^{2}=0.0 \%$; Supplementary Figure 6). 
Table 1

Key characteristics of included studies investigating the association between visual impairment, eye diseases and incident all-cause dementia or key dementia subtypes

\begin{tabular}{|c|c|c|c|c|c|c|c|c|c|c|}
\hline Study & $\begin{array}{l}\text { Country (data } \\
\text { source) }\end{array}$ & Study design & Setting & $\begin{array}{l}\text { Analytic } \\
\text { sample } \\
\text { size } \\
\text { (demen- } \\
\text { tia } \\
\text { cases) } \\
\end{array}$ & $\begin{array}{l}\text { Mean baseline } \\
\text { age (SD) }\end{array}$ & Women, $\%$ & $\begin{array}{l}\text { Race/ } \\
\text { ethnicity* }\end{array}$ & $\begin{array}{l}\text { Mean } \\
\text { follow-up in } \\
\text { years (SD) }\end{array}$ & Exposure & Outcome \\
\hline $\begin{array}{l}\text { Brenowitz } \\
\text { et al. } \\
(2019) \text { [20] }\end{array}$ & $\begin{array}{l}\text { USA (Health } \\
\text { ABC) }\end{array}$ & Cohort & Community & $\begin{array}{l}2,008 \\
\left(378^{\dagger}\right)\end{array}$ & $77.4(2.8) \ddagger$ & $51.8 \ddagger$ & $\begin{array}{l}34.8 \% \\
\text { Black, 65.2\% } \\
\text { White }^{\ddagger}\end{array}$ & $6.5(3.1)^{\dagger}$ & $\begin{array}{l}\text { Visual acuity, } \\
\text { contrast } \\
\text { sensitivity }\end{array}$ & $\begin{array}{l}\text { All-cause } \\
\text { dementia }\end{array}$ \\
\hline $\begin{array}{l}\text { Chen et al. } \\
(2018) \text { [31] }\end{array}$ & $\begin{array}{l}\text { Taiwan } \\
\text { (NHIRD) }\end{array}$ & $\begin{array}{l}\text { Medical } \\
\text { records } \\
\text { cohort }\end{array}$ & Community & $\begin{array}{l}76,585 \\
\left(3,597^{\S}\right)\end{array}$ & $62.1(12.5)$ & 45.3 & $\begin{array}{l}>98 \% \\
\text { Asian }\end{array}$ & $5.0(3.3)$ & $\begin{array}{l}\text { Normal and } \\
\text { high tension } \\
\text { glaucoma }\end{array}$ & $\begin{array}{l}\text { All-cause } \\
\text { dementia, } \\
\text { AD }\end{array}$ \\
\hline $\begin{array}{l}\text { Choi et al. } \\
\text { (2019) [42] }\end{array}$ & $\begin{array}{l}\text { South Korea } \\
\text { (KNHIS- } \\
\text { HEALS) }\end{array}$ & $\begin{array}{l}\text { Medical } \\
\text { records } \\
\text { cohort }\end{array}$ & Community & $\begin{array}{l}308,340 \\
(7,457)\end{array}$ & $60.4(7.8)$ & 48.6 & NR & up to 8 & $\begin{array}{l}\text { Age-related } \\
\text { macular } \\
\text { degenera- } \\
\text { tion }\end{array}$ & $\mathrm{AD}$ \\
\hline $\begin{array}{l}\text { Davies- } \\
\text { Kershaw } \\
\text { et al. } \\
\text { (2018) [21] }\end{array}$ & $\begin{array}{l}\text { United } \\
\text { Kingdom } \\
\text { (ELSA) }\end{array}$ & Cohort & Community & $\begin{array}{l}8,648 \\
(275)\end{array}$ & $66.9^{\dagger}(\mathrm{NR})$ & 55.0 & $\begin{array}{l}97.8 \% \\
\text { White, } 2.2 \% \\
\text { Non-white }\end{array}$ & $11\left(3.7^{\dagger}\right)$ & $\begin{array}{l}\text { Self-reported } \\
\text { vision }\end{array}$ & $\begin{array}{l}\text { All-cause } \\
\text { dementia }\end{array}$ \\
\hline $\begin{array}{l}\text { Ekström \& } \\
\text { Kilander } \\
\text { (2014) [32] }\end{array}$ & $\begin{array}{l}\text { Sweden (not } \\
\text { given) }\end{array}$ & Cohort & Community & $\begin{array}{l}1,123 \\
(174)\end{array}$ & $65-74^{\#}$ & 56.1 & $\begin{array}{l}100 \% \\
\text { White }^{\dagger}\end{array}$ & $14.0(6.4)$ & $\begin{array}{l}\text { Open-angle } \\
\text { glaucoma }\end{array}$ & $\begin{array}{l}\text { All-cause } \\
\text { dementia }\end{array}$ \\
\hline $\begin{array}{l}\text { Exalto et al. } \\
\text { (2014) [45] }\end{array}$ & $\begin{array}{l}\text { USA (KPNC } \\
\text { Diabetes } \\
\text { Registry) }\end{array}$ & $\begin{array}{l}\text { Medical } \\
\text { records } \\
\text { cohort (type } \\
2 \text { diabetes) }\end{array}$ & Community & $\begin{array}{l}29,961 \\
(5,173)\end{array}$ & $70.6(6.8)$ & 46.0 & $\begin{array}{l}63.1 \% \\
\text { White, } 11.4 \% \\
\text { African } \\
\text { American, }\end{array}$ & $6.6(\mathrm{NR})$ & $\begin{array}{l}\text { Diabetic } \\
\text { retinopathy }\end{array}$ & $\begin{array}{l}\text { All-cause } \\
\text { dementia }\end{array}$ \\
\hline $\begin{array}{l}\text { Fischer et al. } \\
\text { (2016) [22] }\end{array}$ & USA (EHLS) & Cohort & Community & $\begin{array}{l}1,884 \\
(187)\end{array}$ & $66.7(8.4)$ & 59.1 & NR & up to 10 & $\begin{array}{l}\text { Contrast } \\
\text { sensitivity }\end{array}$ & $\begin{array}{l}\text { All-cause } \\
\text { dementia }\end{array}$ \\
\hline $\begin{array}{l}\text { Helmer et al. } \\
\text { (2013) [18] }\end{array}$ & $\begin{array}{l}\text { France (3C } \\
\text { Study) }\end{array}$ & Cohort & Community & $812(41)$ & 79.7 (4.3) & 64.7 & NR & & $\begin{array}{l}\text { Open-angle } \\
\text { glaucoma }\end{array}$ & $\begin{array}{l}\text { All-cause } \\
\text { dementia }\end{array}$ \\
\hline $\begin{array}{l}\text { Hwang et al. } \\
\text { (2020) [29] }\end{array}$ & $\begin{array}{l}\text { USA (GEM } \\
\text { Study) }\end{array}$ & $\begin{array}{l}\text { Observational } \\
\text { analysis of } \\
\text { RCT cohort }\end{array}$ & Community & $\begin{array}{l}2,051 \\
(321 / \\
\left.220^{\S} / 86^{\dagger \dagger}\right)\end{array}$ & $78.5(3.1)$ & 44.1 & $\begin{array}{l}97.0 \% \\
\text { White, } \\
3.0 \% \\
\text { Non-white }\end{array}$ & up to 8 & $\begin{array}{l}\text { Self-reported } \\
\text { visual } \\
\text { impairment }\end{array}$ & $\begin{array}{l}\text { All-cause } \\
\text { dementia, } \\
\text { AD, VaD }\end{array}$ \\
\hline
\end{tabular}


Table 1

(Continued)

\begin{tabular}{|c|c|c|c|c|c|c|c|c|c|c|}
\hline Study & $\begin{array}{l}\text { Country (data } \\
\text { source) }\end{array}$ & Study design & Setting & $\begin{array}{l}\text { Analytic } \\
\text { sample } \\
\text { size } \\
\text { (demen- } \\
\text { tia } \\
\text { cases) } \\
\end{array}$ & $\begin{array}{l}\text { Mean baseline } \\
\text { age (SD) }\end{array}$ & Women, $\%$ & $\begin{array}{l}\text { Race/ } \\
\text { ethnicity* }\end{array}$ & $\begin{array}{l}\text { Mean } \\
\text { follow-up in } \\
\text { years (SD) }\end{array}$ & Exposure & Outcome \\
\hline $\begin{array}{l}\text { Keenan et al. } \\
\text { (2015) [38] }\end{array}$ & $\begin{array}{l}\text { United } \\
\text { Kingdom } \\
\text { (NHS HES) }\end{array}$ & $\begin{array}{l}\text { Medical } \\
\text { records } \\
\text { cohort }\end{array}$ & $\begin{array}{l}\text { Secondary } \\
\text { care }\end{array}$ & $\begin{array}{l}2,623,130 \\
\left(30,698^{\S} /\right. \\
\left.29,520^{\dagger \dagger}\right)\end{array}$ & $55+$ & 49.9 & NR & up to 13 & $\begin{array}{l}\text { Primary } \\
\text { open-angle } \\
\text { glaucoma }\end{array}$ & $\mathrm{AD}, \mathrm{VaD}$ \\
\hline $\begin{array}{l}\text { Keenan et al. } \\
\text { (2014) [19] }\end{array}$ & $\begin{array}{l}\text { United } \\
\text { Kingdom } \\
\text { (NHS HES) }\end{array}$ & $\begin{array}{l}\text { Medical } \\
\text { records } \\
\text { cohort }\end{array}$ & $\begin{array}{l}\text { Secondary } \\
\text { care }\end{array}$ & $\begin{array}{l}7,766,857 \\
(\mathrm{NR})\end{array}$ & $50+$ & $61.5^{\ddagger \ddagger}$ & NR & up to 12 & $\begin{array}{l}\text { Age-related } \\
\text { macular } \\
\text { degenera- } \\
\text { tion }\end{array}$ & $\begin{array}{l}\text { All-cause } \\
\text { dementia, } \\
\text { AD }\end{array}$ \\
\hline $\begin{array}{l}\text { Klaver et al. } \\
\text { (1999) [30] }\end{array}$ & $\begin{array}{l}\text { Netherlands } \\
\text { (Rotterdam } \\
\text { Study) }\end{array}$ & Cohort & Community & $\begin{array}{l}1,438 \\
(62)\end{array}$ & $80.7(4.5)$ & 65.4 & Largely White ${ }^{\dagger}$ & $2.1(\mathrm{NR})$ & $\begin{array}{l}\text { Visual acuity, } \\
\text { age-related } \\
\text { macular } \\
\text { degenera- } \\
\text { tion }\end{array}$ & $\mathrm{AD}$ \\
\hline $\begin{array}{l}\text { Kuo et al. } \\
(2020)[33]\end{array}$ & $\begin{array}{l}\text { Taiwan } \\
\text { (NHIRD) }\end{array}$ & $\begin{array}{l}\text { Medical } \\
\text { records } \\
\text { cohort }\end{array}$ & Community & $\begin{array}{l}42,048 \\
(2,304 / \\
183^{\S /} \\
1,784 \dagger)\end{array}$ & $20-100^{\#}$ & 50.0 & $\begin{array}{l}>98 \% \\
\text { Asian }\end{array}$ & up to 16 & $\begin{array}{l}\text { Any } \\
\text { glaucoma, } \\
\text { open-angle } \\
\text { glaucoma, } \\
\text { normal } \\
\text { tension } \\
\text { glaucoma, } \\
\text { angle- } \\
\text { closure } \\
\text { glaucoma }\end{array}$ & $\begin{array}{l}\text { All-cause } \\
\text { dementia, } \\
\text { AD, VaD }\end{array}$ \\
\hline $\begin{array}{l}\text { Lai et al. } \\
\text { (2014) [43] }\end{array}$ & $\begin{array}{l}\text { Taiwan } \\
\text { (NHIRD) }\end{array}$ & $\begin{array}{l}\text { Medical } \\
\text { records } \\
\text { cohort }\end{array}$ & Community & $\begin{array}{l}39,908 \\
(313)\end{array}$ & $71.6(5.25)^{\dagger}$ & 48.1 & $\begin{array}{l}>98 \% \\
\text { Asian }^{\mathbb{I}}\end{array}$ & $7.91(3.32)^{\dagger}$ & Cataract & $\mathrm{AD}$ \\
\hline $\begin{array}{l}\text { Lee et al. } \\
\text { (2019) [34] }\end{array}$ & USA (ACT) & Cohort & Community & $\begin{array}{l}3,877 \\
\left(970^{\dagger} /\right. \\
\left.792^{\S}\right)\end{array}$ & $74.3(6.3)^{\dagger}$ & 58 & $\begin{array}{l}90 \% \\
\text { White }\end{array}$ & $6.6(4.5)^{\dagger}$ & $\begin{array}{l}\text { Glaucoma, } \\
\text { age-related } \\
\text { macular }\end{array}$ & $\begin{array}{l}\text { All-cause } \\
\text { dementia, } \\
\text { AD }\end{array}$ \\
\hline $\begin{array}{l}\text { Lee et al. } \\
(2020) \text { [23] }\end{array}$ & China (EHC) & $\begin{array}{l}\text { Medical } \\
\text { records } \\
\text { cohort }\end{array}$ & Community & $\begin{array}{l}15,576 \\
(1,349)\end{array}$ & $74.5(4.8)$ & 63.8 & $\begin{array}{l}100 \% \\
\text { Asian }\end{array}$ & $\begin{array}{l}5.0 \\
(3.0-6.0)^{\S \S}\end{array}$ & Visual acuity & $\begin{array}{l}\text { All-cause } \\
\text { dementia }\end{array}$ \\
\hline
\end{tabular}




\begin{tabular}{|c|c|c|c|c|c|c|c|c|c|c|}
\hline $\begin{array}{l}\text { Lin et al. } \\
\text { (2014) [39] }\end{array}$ & $\begin{array}{l}\text { Taiwan } \\
\text { (NHIRD) }\end{array}$ & $\begin{array}{l}\text { Medical } \\
\text { records } \\
\text { cohort }\end{array}$ & Community & $\begin{array}{l}19,895 \\
(208)\end{array}$ & $71.3(7.3)$ & 47.1 & $\begin{array}{l}>98 \% \\
\text { Asian }\end{array}$ & $1-8^{\#}$ & $\begin{array}{l}\text { Primary } \\
\text { open-angle } \\
\text { glaucoma }\end{array}$ & $\mathrm{AD}$ \\
\hline $\begin{array}{l}\text { Maruta et al. } \\
(2020) \text { [24] }\end{array}$ & Japan (LTCI) & $\begin{array}{l}\text { Medical } \\
\text { records } \\
\text { cohort }\end{array}$ & Community & $\begin{array}{l}2,190 \\
(1,153)\end{array}$ & $78.9(6.1)$ & 79.4 & $\begin{array}{l}\text { Not } \\
\text { assessed }^{\dagger}\end{array}$ & $8^{\dagger, * *}$ & Visual acuity & $\begin{array}{l}\text { All-cause } \\
\text { dementia }\end{array}$ \\
\hline $\begin{array}{l}\text { Moon et al. } \\
\text { (2018) [40] }\end{array}$ & $\begin{array}{l}\text { South Korea } \\
\text { (KNHIS) }\end{array}$ & $\begin{array}{l}\text { Medical } \\
\text { records } \\
\text { cohort }\end{array}$ & Community & $\begin{array}{l}8,814 \\
(742)\end{array}$ & NR & 47.1 & $\begin{array}{l}100 \% \\
\text { Asian }\end{array}$ & $7.0(\mathrm{NR})^{\S \S}$ & $\begin{array}{l}\text { Primary } \\
\text { open-angle } \\
\text { glaucoma }\end{array}$ & $\mathrm{AD}$ \\
\hline $\begin{array}{l}\text { Naël et al. } \\
\text { (2019) [25] }\end{array}$ & $\begin{array}{l}\text { France (3C } \\
\text { Study) }\end{array}$ & Cohort & Community & $\begin{array}{l}7,736 \\
(882)\end{array}$ & $73.9(5.4)$ & 61.3 & $\begin{array}{l}\text { Not } \\
\text { assessed }^{\dagger}\end{array}$ & $\begin{array}{l}9.1^{\S \S} \\
\left(4.0-11.3^{\dagger}\right)\end{array}$ & $\begin{array}{l}\text { Near visual } \\
\text { acuity, } \\
\text { self-reported } \\
\text { distance } \\
\text { visual } \\
\text { function }\end{array}$ & $\begin{array}{l}\text { All-cause } \\
\text { dementia }\end{array}$ \\
\hline $\begin{array}{l}\text { Nam et al. } \\
\text { (2021) [47] }\end{array}$ & $\begin{array}{l}\text { South Korea } \\
\text { (KNHIS) }\end{array}$ & $\begin{array}{l}\text { Medical } \\
\text { records } \\
\text { cohort }\end{array}$ & Community & $\begin{array}{l}185,036 \\
(14,727 / \\
10,965^{\S} / \\
\left.1,788^{\dagger \dagger}\right)\end{array}$ & $63.4(10.1)$ & 56.4 & $\begin{array}{l}\text { 100\% } \\
\text { Asian }{ }^{\text {III }}\end{array}$ & $6.6(1.4)$ & $\begin{array}{l}\text { Retinal vein } \\
\text { occlusion }\end{array}$ & $\begin{array}{l}\text { All-cause } \\
\text { dementia, } \\
\text { AD, VaD }\end{array}$ \\
\hline $\begin{array}{l}\text { Ou et al. } \\
\text { (2012) [35] }\end{array}$ & $\begin{array}{l}\text { USA } \\
\text { (Medicare) }\end{array}$ & $\begin{array}{l}\text { Medical } \\
\text { records } \\
\text { cohort }\end{array}$ & Community & $\begin{array}{l}126,650 \\
(40,528 / \\
\left.21,531^{\S}\right)\end{array}$ & $68+$ & 65 & $\begin{array}{l}86.9 \% \text { White, } \\
11 \% \text { Black, } \\
2.1 \% \text { Other }\end{array}$ & up to 14 & $\begin{array}{l}\text { Open-angle } \\
\text { glaucoma }\end{array}$ & $\begin{array}{l}\text { All-cause } \\
\text { dementia, } \\
\text { AD }\end{array}$ \\
\hline $\begin{array}{l}\text { Paik et al. } \\
(2020)[28]\end{array}$ & $\begin{array}{l}\text { South Korea } \\
\text { (KNHIS) }\end{array}$ & $\begin{array}{l}\text { Medical } \\
\text { records } \\
\text { cohort }\end{array}$ & Community & $\begin{array}{l}6,029,657 \\
(165,293 / \\
123,497^{\S} / \\
\left.20,678^{\dagger \dagger}\right)\end{array}$ & $54.2(10.5)$ & 50.7 & $\begin{array}{l}100 \% \\
\text { Asian }^{\text {IIII }}\end{array}$ & $5.75(0.92)$ & Visual acuity & $\begin{array}{l}\text { All-cause } \\
\text { dementia, } \\
\text { AD, VaD }\end{array}$ \\
\hline $\begin{array}{l}\text { Rodill et al. } \\
\text { (2018) [44] }\end{array}$ & USA (KPNC) & $\begin{array}{l}\text { Medical } \\
\text { records } \\
\text { cohort (type } \\
1 \text { diabetes) }\end{array}$ & Community & $\begin{array}{l}3,742 \\
(182)\end{array}$ & $56.1(8.5)$ & & $\begin{array}{l}\text { 79.0\% White, } \\
5.0 \% \text { Black, } \\
5.6 \% \\
\text { Hispanic, } 3.9\end{array}$ & $6.2(5.3)$ & $\begin{array}{l}\text { Diabetic } \\
\text { retinopathy }\end{array}$ & $\begin{array}{l}\text { All-cause } \\
\text { dementia }\end{array}$ \\
\hline $\begin{array}{l}\text { Rogers \& } \\
\text { Langa } \\
\text { (2010) [26] }\end{array}$ & USA (HRS) & Cohort & Community & $\begin{array}{l}625 \\
(168)\end{array}$ & $71+$ & 61.3 & $\begin{array}{l}7.9 \% \text { Non- } \\
\text { Caucasian }\end{array}$ & $8.5(2.4)$ & $\begin{array}{l}\text { Self-reported } \\
\text { vision }\end{array}$ & $\begin{array}{l}\text { All-cause } \\
\text { dementia }\end{array}$ \\
\hline $\begin{array}{l}\text { Schrijvers } \\
\text { et al. (2012) } \\
\text { [46] }\end{array}$ & $\begin{array}{l}\text { Netherlands } \\
\text { (Rotterdam } \\
\text { Study) }\end{array}$ & Cohort & Community & $\begin{array}{l}6,078 \\
(735 / \\
\left.583^{\S} / 80^{\dagger \dagger}\right)\end{array}$ & $68.3(8.4)$ & 59 & NR & $11.4(\mathrm{NR})$ & $\begin{array}{l}\text { Diabetic } \\
\text { retinopathy }\end{array}$ & $\begin{array}{l}\text { All-cause } \\
\text { dementia, } \\
\text { AD, VaD }\end{array}$ \\
\hline
\end{tabular}


Table 1

\begin{tabular}{|c|c|c|c|c|c|c|c|c|c|c|}
\hline$\overline{\text { Study }}$ & $\begin{array}{l}\text { Country (data } \\
\text { source) }\end{array}$ & Study design & Setting & $\begin{array}{l}\text { Analytic } \\
\text { sample } \\
\text { size } \\
\text { (demen- } \\
\text { tia } \\
\text { cases) }\end{array}$ & $\begin{array}{l}\text { Mean baseline } \\
\text { age (SD) }\end{array}$ & Women, $\%$ & $\begin{array}{l}\text { Race/ } \\
\text { ethnicity* }\end{array}$ & $\begin{array}{l}\text { Mean } \\
\text { follow-up in } \\
\text { years (SD) }\end{array}$ & Exposure & Outcome \\
\hline $\begin{array}{l}\text { Su et al. } \\
(2016) \text { [36] }\end{array}$ & $\begin{array}{l}\text { Taiwan } \\
\text { (NHIRD) }\end{array}$ & $\begin{array}{l}\text { Medical } \\
\text { records } \\
\text { cohort }\end{array}$ & Community & $\begin{array}{l}32,545 \\
(1,601)\end{array}$ & $59.2(17.2)$ & 54.5 & $\begin{array}{l}>98 \% \\
\text { Asian }^{\mathbb{I I}}\end{array}$ & $6.3(3.1)^{\dagger}$ & $\begin{array}{l}\text { Glaucoma, } \\
\text { primary } \\
\text { angle- } \\
\text { closure } \\
\text { glaucoma, } \\
\text { primary } \\
\text { open-angle } \\
\text { glaucoma }\end{array}$ & $\begin{array}{l}\text { All-cause } \\
\text { dementia }\end{array}$ \\
\hline $\begin{array}{l}\text { Tran et al. } \\
\text { (2020) [27] }\end{array}$ & USA (WHI) & $\begin{array}{l}\text { Observational } \\
\text { analysis of } \\
\text { RCT cohort }\end{array}$ & Community & $\begin{array}{l}1,061 \\
(42)\end{array}$ & $73.8(3.7)$ & 100 & $\begin{array}{l}90.4 \% \text { White, } \\
6.0 \% \text { Black, } \\
1.4 \% \\
\text { Hispanic, } \\
0.3 \% \\
\text { American } \\
\text { Indian, } 0.8 \% \\
\text { Asian/Pacific } \\
\text { Islander, } 1.0 \% \\
\text { Other }\end{array}$ & $3.8(1.8)$ & $\begin{array}{l}\text { Visual acuity, } \\
\text { self-reported } \\
\text { visual } \\
\text { impairment }\end{array}$ & $\begin{array}{l}\text { All-cause } \\
\text { dementia }\end{array}$ \\
\hline $\begin{array}{l}\text { Tsai et al. } \\
\text { (2015) [41] }\end{array}$ & $\begin{array}{l}\text { Taiwan } \\
\text { (NHIRD) }\end{array}$ & $\begin{array}{l}\text { Medical } \\
\text { records } \\
\text { cohort }\end{array}$ & Community & $\begin{array}{l}29,958 \\
(1,589)\end{array}$ & $74.5(5.8)$ & 47 & $\begin{array}{l}>98 \% \\
\text { Asian }\end{array}$ & $4.4(2.5)$ & $\begin{array}{l}\text { Age-related } \\
\text { macular } \\
\text { degenera- } \\
\text { tion }\end{array}$ & $\begin{array}{l}\text { All-cause } \\
\text { dementia }\end{array}$ \\
\hline $\begin{array}{l}\text { Xiao et al. } \\
\text { (2020) [37] }\end{array}$ & China (SAS) & Cohort & Community & $\begin{array}{l}1,659 \\
\left(168 / 124^{\S}\right)\end{array}$ & $71.5(7.4)$ & & NR & $5.2(\mathrm{NR})$ & $\begin{array}{l}\text { Glaucoma, } \\
\text { cataract }\end{array}$ & $\begin{array}{l}\text { All-cause } \\
\text { dementia, } \\
\text { AD }\end{array}$ \\
\hline
\end{tabular}

ACT, Adult Changes in Thought; AD, Alzheimer's disease; AMD, age-related macular degeneration; DNHR, Danish National Hospital Register; DPCR, Danish Psychiatric Central Register; DR, diabetic retinopathy; EHC, Elderly Health Centres; EHLS, Epidemiology of Hearing Loss Study; Health ABC, Health, Aging, and Body Composition; GEM, Ginkgo Evaluation of Memory; HRS, Health and Retirement Study; KNHIS-HEALS, Korean National Health Insurance Service - Health Screening Cohort; KPNC, Kaiser Permanente Northern California; LTCI, Long-term Care Insurance; NA, not applicable; NHIRD, National Health Insurance Database; NHS HES, National Health Service Hospital Episode Statistics; NR, not reported; PACG, primary angle-closure glaucoma; POAG, primary open-angle glaucoma; RCT, randomized controlled trial; SAS, Shanghai Aging Study; VaD, vascular dementia; WHI, Women's Health Initiative; 3C, Three-City; ${ }^{*}$ Percentages may not sum up to $100 \%$ due to rounding; ${ }^{\dagger}$ Additional information provided by the authors; ${ }^{\ddagger}$ Reported for the sample of 1,$810 ;{ }^{8} \mathrm{AD} ;{ }^{\mathbb{T}}$ As described in Kuo et al. [33]; ${ }^{\sharp}$ Range; $* *$ All participants followed for the reported period; ${ }^{\dagger \dagger} \mathrm{VaD}$; ${ }^{\ddagger \dagger}$ Reported for the sample of 65,894 with age-related macular degeneration; ${ }^{\S}$ Median (interquartile range); ${ }^{\mathbb{4}}$ As As described in Moon et al. [40]. 


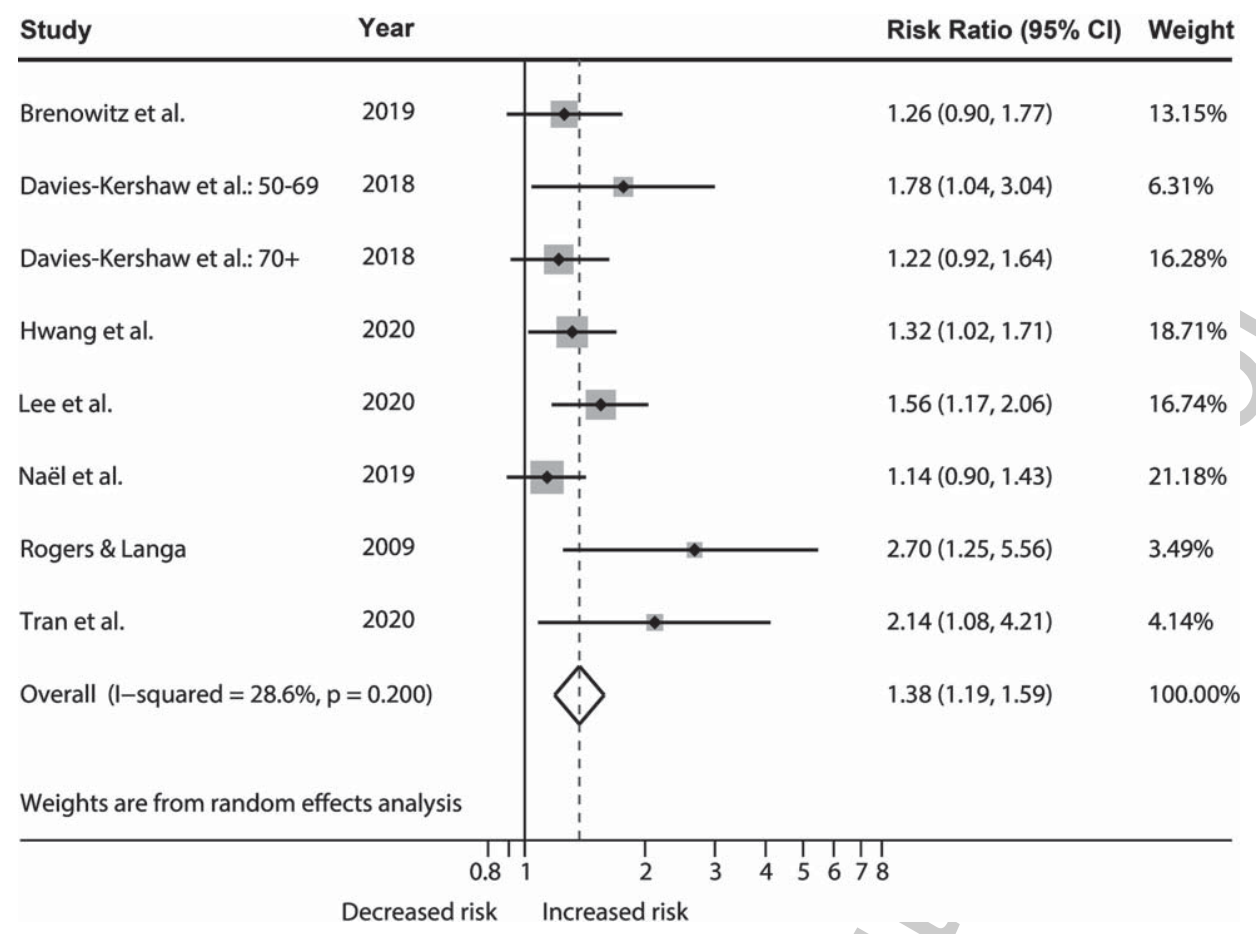

Fig. 2. Meta-analysis of risk ratios of at least mild visual impairment compared to no visual impairment on incident all-cause dementia. CI, confidence interval.
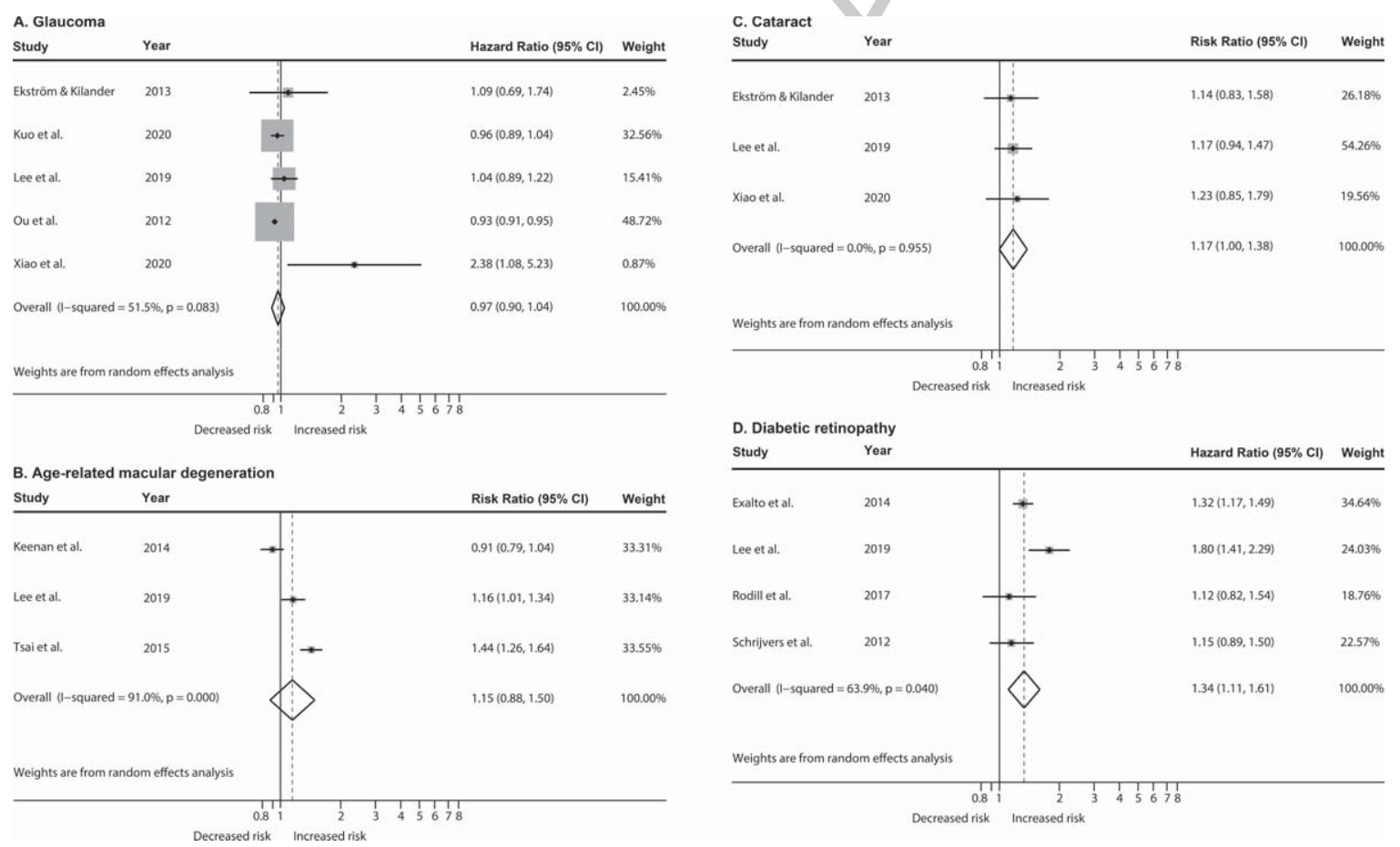

Fig. 3. Meta-analysis of risk ratios of eye disease compared to no given eye disease on incident all-cause dementia. CI, confidence interval. 


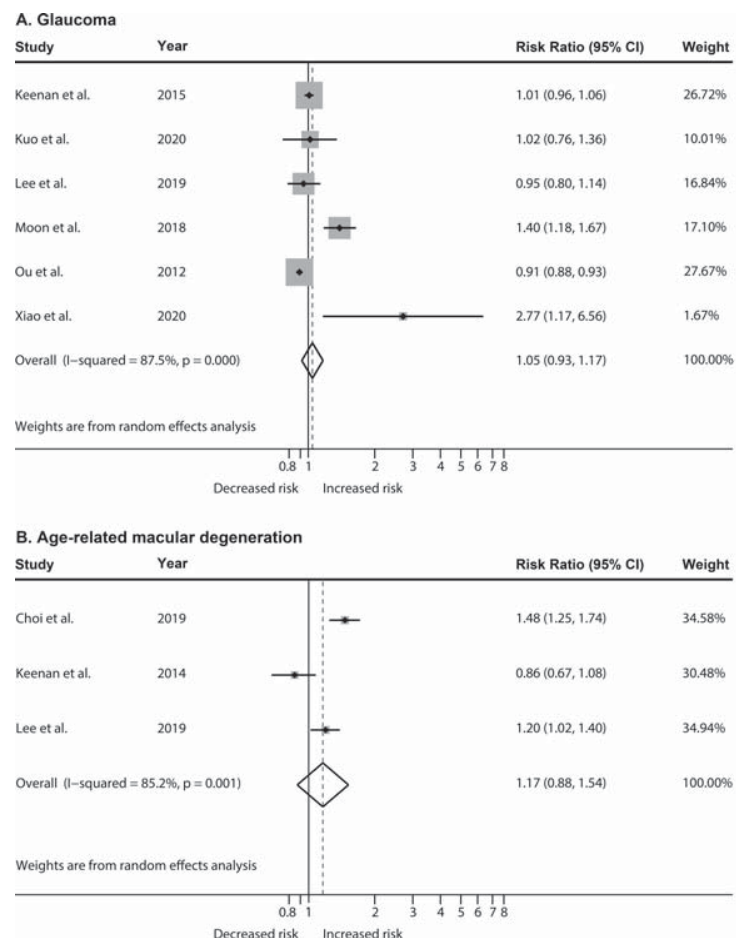

participants, 31,648 AD cases, pooled adjusted $\mathrm{RR}=1.23$, 95\% CI: $0.94-1.60, p=0.13, \mathrm{I}^{2}=87.7 \%$, Supplementary Figure 8).

Two cohort studies investigated the association between glaucoma and incident $\mathrm{VaD}[33,38]$ (Supplementary Table 4 ). The pooled estimate suggested no association between open-angle glaucoma and $\mathrm{VaD}(2,665,178$ participants, 31,304 VaD cases, pooled adjusted $\mathrm{RR}=0.97,95 \% \mathrm{CI}: 0.73-1.27$, $p=0.81, \mathrm{I}^{2}=90.7 \%$; Supplementary Figure 7).

\section{Age-related macular degeneration}

Five cohort studies reported the association between AMD and incident all-cause dementia [19, $34,41](n=3)$ and/or AD [19, 30, 34, 42] $(n=4$; Supplementary Table 4). Pooled estimates of three studies $[19,34,41]$ provided little evidence of an association between AMD and all-cause dementia (7,800,692 participants, $>2,559$ dementia cases [exact number cannot be determined], pooled adjusted $\mathrm{RR}=1.15$, 95\% CI: $0.88-1.50, p=0.30, \mathrm{I}^{2}=91.0 \%$, Fig. 3 ) or $\operatorname{AD}(8,079,074$ participants, $>8,249$ AD cases [exact number cannot be determined], pooled adjusted $\mathrm{RR}=1.17,95 \%$ CI: $0.88-1.54, p=0.28, \mathrm{I}^{2}=85.2 \%$, Fig. 4).
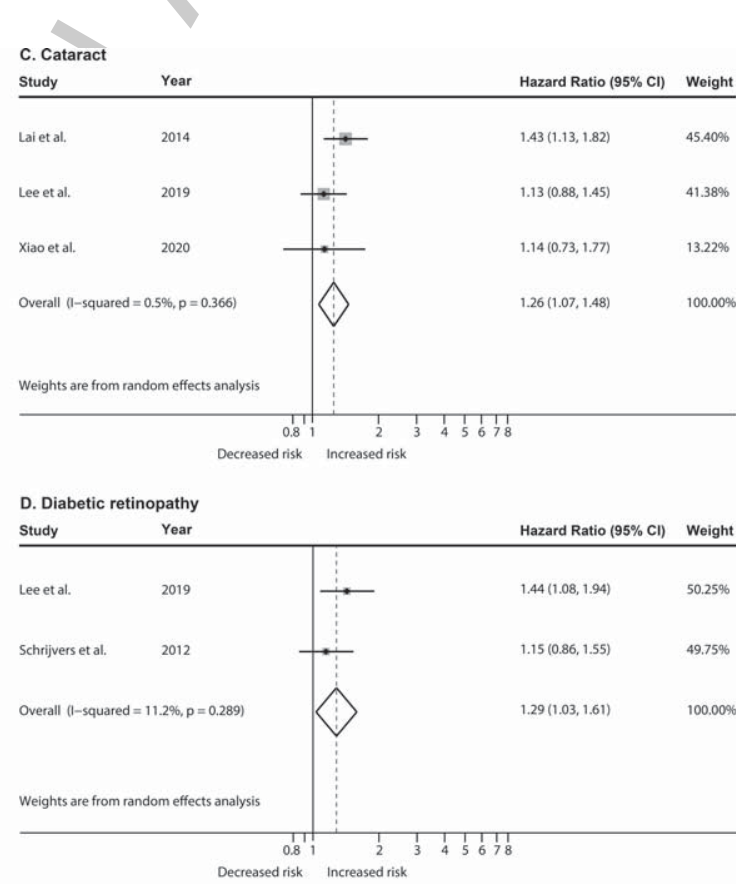

Fig. 4. Meta-analysis of risk ratios of eye disease compared to no given eye disease on incident Alzheimer's disease. CI, confidence interval. 


\section{Cataract}

Four cohort studies investigated the association between cataracts and incident all-cause dementia $[32,34,37](n=3)$ and/or $\mathrm{AD}[34,37,43](n=$ 3; Supplementary Table 5). The pooled estimates suggested an increased risk of all-cause dementia (6,659 participants, 1,312 dementia cases, pooled adjusted $\mathrm{RR}=1.17,95 \% \mathrm{CI}: 1.00-1.38, p=0.06, \mathrm{I}^{2}$ $=0.0 \%$, Fig. 3$)$ and of $\operatorname{AD~}(45,444$ participants, 1,229 $\mathrm{AD}$ cases, pooled $\mathrm{HR}=1.26,95 \% \mathrm{CI}: 1.07-1.48$, $p=0.005, \mathrm{I}^{2}=0.5 \%$, Fig. 4) in those with cataract compared to no cataract.

\section{Diabetic retinopathy}

Four studies based on one type 1 diabetic cohort [44], one type 2 diabetic cohort [45] and two cohorts including non-diabetic participants $[34,46]$ investigated the association between diabetic retinopathy and incident all-cause dementia with one [46] and two $[34,46]$ studies, respectively, also investigating incident $\mathrm{VaD}$ and $\mathrm{AD}$. The pooled estimate of four [34, 44-46] and two studies [34, 46], respectively, indicated an increased risk of all-cause dementia (43,658 participants, 7,060 dementia cases, pooled adjusted $\mathrm{HR}=1.34,95 \% \mathrm{CI}: 1.11-1.61, p=0.002$, $\mathrm{I}^{2}=63.9 \%$, Fig. 3) and AD (9,955 participants, 1,375 AD cases, pooled adjusted $\mathrm{HR}=1.29,95 \%$ CI: $1.03-1.61, p=0.03, \mathrm{I}^{2}=11.2 \%$, Fig. 4 ) in those with diabetic retinopathy compared to no retinopathy. Only one study [46] (6,078 participants, $80 \mathrm{VaD}$ cases) investigated diabetic retinopathy and $\mathrm{VaD}$; this provided little evidence of an association $(\mathrm{HR}=0.90$, 95\% CI: 0.39-2.11).

\section{Other eye diseases}

One study [47] investigating the association with retinal vein occlusion using medical records suggested an increased risk of incident all-cause dementia $(185,036$ participants, 14,727 dementia cases, HR =1.16, 95\% CI: 1.12-1.21), AD (185,036 participants, $10,965 \mathrm{AD}$ cases, $\mathrm{HR}=1.15,95 \% \mathrm{CI}$ : 1.11-1.20), and $\operatorname{VaD}(185,036$ participants, 1,788 $\mathrm{VaD}$ cases, $\mathrm{HR}=1.24,95 \% \mathrm{CI}: 1.12-1.37)$ compared to no retinal vein occlusion (Supplementary Table 7).

\section{Additional analyses}

Associations remained similar when we repeated analyses with only studies that received a global risk of bias rating of moderate or strong. Pooled estimates for these analyses are provided in Supplementary Results.

\section{DISCUSSION}

Results of our systematic review and meta-analyses indicate that VI based on visual acuity was associated with incident all-cause dementia, AD, and $\mathrm{VaD}$. The association with all-cause dementia was robust to exclusion of studies using self-reported VI and studies with a weak global risk of bias rating. However, there was no association between VI measured using contrast sensitivity and dementia. Diabetic retinopathy was also associated with an increased dementia and AD risk. There was weaker evidence for an increased dementia and AD risk in those with cataract. We found no evidence of an association between glaucoma or AMD and risk of dementia or key dementia subtypes.

Our findings are in line with results of a recently published meta-analysis reporting similar pooled estimates of the association between visual impairment and incident dementia or cognitive impairment [7]. However, we applied more stringent inclusion and exclusion criteria (e.g., exclusion of studies investigating color vision deficiency, studies including overlapping data and non-peer-reviewed publications) potentially resulting in more conservative estimates. Nevertheless, despite the different criteria for study selection, the findings from both metaanalyses provide consistent evidence that VI is associated with a $38 \%-47 \%$ increased risk of dementia.

In our meta-analysis, the exposure was defined as at least mild VI, so when comparing to no VI the strength of the relationship between moderate or severe VI and dementia might have been underestimated. For example, Paik and colleagues [28] reported stronger associations for moderate $\mathrm{VI}(\mathrm{HR}=$ 1.74, 95\% CI: 1.70-1.78) and severe VI/blindness $(\mathrm{HR}=1.75,95 \% \mathrm{CI}: 1.71-1.79)$ than for mild VI $(\mathrm{HR}=1.45$, 95\% CI: $1.42-1.48)$ compared to normal vision. Some studies included in the systematic review also found higher dementia risk in those with more severe VI, but only contributed results for 'mild' VI to the pooled estimates [21, 23, 25].

The association between VI and dementia risk could be mediated by other dementia risk factors, e.g., social isolation, depressive symptom, and physical inactivity [3]. Findings from the Three-City study [25] and the Canadian Longitudinal Study on Aging 
[48] suggest that engagement in cognitively stimulating activities and social engagement only weakly mediate this association. However, the prevalence of depression is higher in those with VI compared to normal vision [49] and dementia risk increased only in those with depressive symptoms in the ThreeCity study [25]. Physical inactivity is an established dementia risk factor [3] also related to VI where VI may lead to decreased physical activity whereas being physically active may protect against VI [50]. Nevertheless, the relationships are complex as the proposed mediators may increase dementia risk, be an early sign of dementia [3], or be bi-directionally related with VI [50, 51].

VI often co-occurs with hearing impairment, another form of sensory deprivation which has recently been highlighted as a potential modifiable risk factor for dementia [3]. Both sensory impairments may share common pathways [52] and the risk of dementia might be greater in the presence of both VI and hearing impairment. Two [27, 29] of the studies included in the systematic review investigated both impairments. Hwang and colleagues [29] found dual sensory impairment was associated with a much higher dementia risk $(\mathrm{HR}=1.86,95 \%$ CI:1.25-2.76) than single impairment $(\mathrm{HR}=1.11$, 95\% CI:0.86-1.44) compared to no sensory impairment. In analyses stratified by self-reported hearing loss, Tran and colleagues [27] reported a stronger association between VI and dementia risk in those with hearing loss. However, only two additional studies [23, 25] investigating VI adjusted their analyses for hearing impairment. Further studies clarifying whether VI and hearing impairment independently or interactively increase the risk of dementia are therefore warranted

Glaucoma and AD are both neurodegenerative diseases and share pathogenic mechanisms, such as axonal degeneration, altered levels of $A \beta$ and tau proteins, cerebrospinal fluid circulatory failure, and autophagy [53]. Therefore, glaucoma could either increase the risk of dementia due to VI or commonly co-occur due to these shared mechanisms. However, our findings are similar to a previous meta-analysis of five prospective studies [8] which suggested no association between glaucoma and AD.

Shared risk factors (e.g., smoking, hypertension, obesity) and cellular processes (e.g., oxidative stress, chronic inflammation, ubiquitination) have been also suggested for AMD and AD [54]. However, neither results of our meta-analysis nor findings of a previous meta-analysis when restricted to prospective studies
[9] provide evidence for an association between AMD and dementia risk. Common risk factors (e.g., age, smoking, low socioeconomic status) may also explain an increased risk of all-cause dementia and $\mathrm{AD}$ in those with cataract in our meta-analyses [55].

Diabetes, often accompanied by damage to the retina, is an established risk factor for all-cause dementia $(\mathrm{RR}=1.43,95 \% \mathrm{CI}: 1.33-1.53), \mathrm{AD}$ $(\mathrm{RR}=1.43,95 \% \mathrm{CI}: 1.25-1.62)$ and $\mathrm{VaD}(\mathrm{RR}=1.91$, 95\% CI: 1.61-2.25) [56]. Our meta-analyses yielded similar estimates for the association of diabetic retinopathy with all-cause dementia or AD. Therefore, common metabolic and vascular pathways (e.g., hyperglycemia, hyperlipidemia, hypertension) underling both dementia and diabetic retinopathy are possible. Retinal microvascular changes may indicate cerebral vascular disease given that retinal vessels are similar to cerebral microvasculature and have been suggested as potential dementia biomarkers $[10,57]$.

Included studies differed considerably in exposure ascertainment. Most studies of VI used visual acuity tests or relied on self-report whereas most studies of eye diseases were based on medical records. Eye diseases captured through medical records would be expected to be treated and therefore could have biased the results towards the null. Indeed, a systematic review and meta-analysis of cataract surgery indicated an improvement in cognitive function and reduction of depressive symptoms after cataract treatment [58]. Moreover, some cases of eye diseases and dementia may not be recorded in medical records, also biasing the results towards the null. Furthermore, in studies focusing on specific eye diseases, the comparison group will include individuals with other eye diseases (unless explicitly excluded). Again, this could bias the results towards the null, if the hypothesis is that VI increases the risk of dementia, rather than specific conditions. The length of follow-up of studies included in our systematic review ranged up to 16 years [33] but changes in the brain may occur even 20 years before the onset of dementia symptoms [59]. Moreover, not all included studies were specifically designed to investigate the association between VI and dementia, and generally there was high degree of heterogeneity between the studies reflecting methodological differences, e.g., in sample selection, adjustment strategy, exposure, and outcome ascertainment. Half of the studies included in meta-analyses received a risk of bias rating of weak. Therefore, further well-designed studies are 
needed to investigate the association between VI, eye diseases, and risk of dementia and key dementia subtypes. Our search strategy was limited to three databases and focused on VI in general and common eye diseases, not including contrast sensitivity specifically or rarer eye diseases. Therefore, we cannot exclude the possibility that not all relevant studies were captured. Finally, the observational nature of the studies included in the review limit any causal inferences on the associations observed.

In conclusion, this systematic review and metaanalysis of VI and its major causes provides evidence for the association between VI and increased dementia risk. Diabetic retinopathy and cataract but not glaucoma and AMD were also associated with increased dementia risk. Diagnosing VI may help identify those at risk of developing dementia. Given that VI is highly prevalent and most causes of VI are treatable, the potential for dementia prevention of early interventions to reduce VI warrants further investigation.

\section{ACKNOWLEDGMENTS}

We thank all authors of original studies who supported our work by providing additional data and/or clarifications.

This study was supported by the Nicolaus and Margrit Langbehn Foundation (to E.K. and U.T.), a Moorfields Eye Charity Career Development Fellowship and a UK Research and Innovation Future Leaders Fellowship (to A:P:K.), the National Institute for Health Research (NIHR) Applied Research Collaboration (ARC) South West Peninsula (to D.J.L. and O.C.U.), Alzheimer's Research UK, National Health and Medical Research Council (NHMRC), JP Moulton Foundation, National Institute on Aging/National Institutes of Health (RF1AG055654), Alan Turing Institute/Engineering and Physical Sciences Research Council (EP/N510129/1; to D.J.L.), the Federal Ministry of Education and Research (BMBF) and the Federal Joint Committee (G-BA) Innovation Fund (to U.T). The views expressed are those of the author(s) and not necessarily those of the National Health Service, the National Institute for Health Research, or the Department of Health and Social Care or other funders.

Authors' disclosures available online (https:// www.j-alz.com/manuscript-disclosures/21-0250r2).

\section{SUPPLEMENTARY MATERIAL}

The supplementary material is available in the electronic version of this article: https://dx.doi.org/ 10.3233/JAD-210250.

\section{REFERENCES}

[1] Albers MW, Gilmore GC, Kaye J, Murphy C, Wingfield A, Bennett DA, Boxer AL, Buchman AS, Cruickshanks KJ, Devanand DP, Duffy CJ, Gall CM, Gates GA, Granholm A-C, Hensch T, Holtzer R, Hyman BT, Lin FR, McKee AC, Morris JC, Petersen RC, Silbert LC, Struble RG, Trojanowski JQ, Verghese J, Wilson DA, Xu S, Zhang LI (2015) At the interface of sensory and motor dysfunctions and Alzheimer's disease. Alzheimers Dement 11, 70-98.

[2] Burton MJ, Ramke J, Marques AP, Bourne RRA, Congdon N, Jones I, Ah Tong BAM, Arunga S, Bachani D, Bascaran C, Bastawrous A, Blanchet K, Braithwaite T, Buchan JC, Cairns J, Cama A, Chagunda M, Chuluunkhuu C, Cooper A, Crofts-Lawrence J, Dean WH, Denniston AK, Ehrlich JR, Emerson PM, Evans JR, Frick KD, Friedman DS, Furtado JM, Gichangi MM, Gichuhi S, Gilbert SS, Gurung R, Habtamu E, Holland P, Jonas JB, Keane PA, Keay L, Khanna RC, Khaw PT, Kuper H, Kyari F, Lansingh VC, Mactaggart I, Mafwiri MM, Mathenge W, McCormick I, Morjaria P, Mowatt L, Muirhead D, Murthy GVS, Mwangi N, Patel DB, Peto T, Qureshi BM, Salomão SR, Sarah V, Shilio BR, Solomon AW, Swenor BK, Taylor HR, Wang N, Webson A, West SK, Wong TY, Wormald R, Yasmin S, Yusufu M, Silva JC, Resnikoff S, Ravilla T, Gilbert CE, Foster A, Faal HB (2021) The Lancet Global Health Commission on Global Eye Health: Vision beyond 2020. Lancet Glob Health 9, e489-e551.

[3] Livingston G, Huntley J, Sommerlad A, Ames D, Ballard C, Banerjee S, Brayne C, Burns A, Cohen-Mansfield J, Cooper C, Costafreda SG, Dias A, Fox N, Gitlin LN, Howard R, Kales HC, Kivimäki M, Larson EB, Ogunniyi A, Orgeta V, Ritchie K, Rockwood K, Sampson EL, Samus Q, Schneider LS, Selbæk G, Teri L, Mukadam N (2020) Dementia prevention, intervention, and care: 2020 report of the Lancet Commission. Lancet 396, 413-446.

[4] Livingston G, Sommerlad A, Orgeta V, Costafreda S, Huntley J, Ames D, Ballard C, Banerjee S, Burns A, CohenMansfield J, Fox N, Gitlin L, Howard R, Kales H, Larson E, Ritchie K, Rockwood K, Sampson E, Mukadam N (2017) Dementia prevention, intervention, and care. Lancet 390, 2673-2734.

[5] World Health Organisation (2013) Universal Eye Health: A global action plan 2014-2019. https://www. who.int/blindness/actionplan/en/. Accessed on July 24, 2020.

[6] Bourne RRA, Flaxman SR, Braithwaite T, Cicinelli MV, Das A, Jonas JB, Keeffe J, Kempen JH, Leasher J, Limburg H, Naidoo K, Pesudovs K, Resnikoff S, Silvester A, Stevens GA, Tahhan N, Wong TY, Taylor HR (2017) Magnitude, temporal trends, and projections of the global prevalence of blindness and distance and near vision impairment: A systematic review and meta-analysis. Lancet Glob Health 5, e888-e897.

[7] Shang X, Zhu Z, Wang W, Ha J, He M (2021) The association between vision impairment and incidence 
of dementia and cognitive impairment: A systematic review and meta-analysis. Ophthalmology, doi.org/ 10.1016/j.ophtha.2020.12.029

[8] Xu XH, Zou JY, Geng W, Wang AY (2019) Association between glaucoma and the risk of Alzheimer's disease: A systematic review of observational studies. Acta Ophthalmol 97, 665-671.

[9] Rong SS, Lee BY, Kuk AK, Yu XT, Li SS, Li J, Guo Y, Yin Y, Osterbur DL, Yam JCS, Cheung CY, Chen LJ, Wong TY, Ng DS (2019) Comorbidity of dementia and age-related macular degeneration calls for clinical awareness: A metaanalysis. Br J Ophthalmol 103, 1777-1783.

[10] Heringa SM, Bouvy WH, van den Berg E, Moll AC, Kappelle LJ, Biessels GJ (2013) Associations between retinal microvascular changes and dementia, cognitive functioning, and brain imaging abnormalities: A systematic review. J Cereb Blood Flow Metab 33, 983-995.

[11] Centre for Reviews and Dissemination (2009) Systematic reviews: CRD's guidance for undertaking reviews in health care, University of York, York, UK.

[12] Chou R, Dana T, Bougatsos C (2009) Screening for Visual Impairment in Older Adults: Systematic Review to Update the 1996 U.S. Preventive Services Task Force Recommendation, Agency for Healthcare Research and Quality (US), Report No.; 09-05135-EF-1, Rockville MD.

[13] Kuźma E, Lourida I, Moore SF, Levine DA, Ukoumunne OC, Llewellyn DJ (2018) Stroke and dementia risk: A systematic review and meta-analysis. Alzheimers Dement 14, 1416-1426.

[14] Thomas BH, Ciliska D, Dobbins M, Micucci S (2004) A process for systematically reviewing the literature: Providing the research evidence for public health nursing interventions. Worldviews Evid Based Nurs 1, 176-184.

[15] Harris RJ, Deeks JJ, Altman DG, Bradburn MJ, Harbord RM, Sterne JAC (2008) Metan: Fixed- and random-effects meta-analysis. Stata J 8, 3-28.

[16] Higgins JP, Thompson SG, Deeks JJ, Altman DG (2003) Measuring inconsistency in meta-analyses. BMJ 327, 557-560.

[17] Higgins JPT, Thomas J, Chandler J, Cumpston M, Li T, Page MJ, Welch VA (2019) Cochrane Handbook for Systematic Reviews of Interventions version 6.0 (updated July 2019), https://www.who.int/blindness/actionplan/en/. Accessed on October 3, 2020.

[18] Helmer C, Malet F, Rougier MB, Schweitzer C, Colin J, Delyfer MN, Korobelnik JF, Barberger-Gateau P, Dartigues JF, Delcourt C (2013) Is there a link between open-angle glaucoma and dementia? The Three-City-Alienor cohort. Ann Neurol 74, 171-179.

[19] Keenan TD, Goldacre R, Goldacre MJ (2014) Associations between age-related macular degeneration, Alzheimer disease, and dementia: Record linkage study of hospital admissions. JAMA Ophthalmol 132, 63-68.

[20] Brenowitz WD, Kaup AR, Lin FR, Yaffe K (2019) Multiple sensory impairment is associated with increased risk of dementia among black and white older adults. J Gerontol A Biol Sci Med Sci 74, 890-896.

[21] Davies-Kershaw HR, Hackett RA, Cadar D, Herbert A, Orrell M, Steptoe A (2018) Vision impairment and risk of dementia: Findings from the English Longitudinal Study of Ageing. J Am Geriatr Soc 66, 1823-1829.

[22] Fischer ME, Cruickshanks KJ, Schubert CR, Pinto AA, Carlsson CM, Klein BE, Klein R, Tweed TS (2016) Age-related sensory impairments and risk of cognitive impairment. J Am Geriatr Soc 64, 1981-1987.
[23] Lee ATC, Richards M, Chan WC, Chiu HFK, Lee RSY, Lam LCW (2020) Higher dementia incidence in older adults with poor visual acuity. J Gerontol A Biol Sci Med Sci 75, 2162-2168.

[24] Maruta M, Tabira T, Sagari A, Miyata H, Yoshimitsu K, Han G, Yoshiura K, Matsuo T, Kawagoe M (2020) Impact of sensory impairments on dementia incidence and symptoms among Japanese older adults. Psychogeriatrics 20, 262-270.

[25] Naël V, Peres K, Dartigues JF, Letenneur L, Amieva H, Arleo A, Scherlen AC, Tzourio C, Berr C, Carriere I, Delcourt C, Helmer C (2019) Vision loss and 12-year risk of dementia in older adults: The 3C cohort study. Eur J Epidemiol 34, 141-152.

[26] Rogers MA, Langa KM (2010) Untreated poor vision: A contributing factor to late-life dementia. Am J Epidemiol 171, 728-735.

[27] Tran EM, Stefanick ML, Henderson VW, Rapp SR, Chen JC, Armstrong NM, Espeland MA, Gower EW, Shadyab AH, Li W, Stone KL, Pershing S (2020) Association of visual impairment with risk of incident dementia in a Women's Health Initiative Population. JAMA Ophthalmol 138, 624-633.

[28] Paik J-S, Ha M, Jung YH, Kim G-H, Han K-D, Kim H-S, Lim DH, Na K-S (2020) Low vision and the risk of dementia: A nationwide population-based cohort study. Sci Rep 10, 9109.

[29] Hwang PH, Longstreth WT, Jr., Brenowitz WD, Thielke SM, Lopez OL, Francis CE, DeKosky ST, Fitzpatrick AL (2020) Dual sensory impairment in older adults and risk of dementia from the GEM Study. Alzheimers Dement 12 , e12054.

[30] Klaver CC, Ott A, Hofman A, Assink JJ, Breteler MM, de Jong PT (1999) Is age-related maculopathy associated with Alzheimer's disease? The Rotterdam Study. Am J Epidemiol 150, 963-968.

[31] Chen YY, Lai YJ, Yen YF, Shen YC, Wang CY, Liang CY, Lin KH, Fan LW (2018) Association between normal tension glaucoma and the risk of Alzheimer's disease: A nationwide population-based cohort study in Taiwan. BMJ Open 8, e022987.

[32] Ekstrom C, Kilander L (2014) Pseudoexfoliation and Alzheimer's disease: A population-based 30-year follow-up study. Acta Ophthalmol 92, 355-358.

[33] Kuo FH, Chung JF, Hsu MY, Lee CY, Huang JY, Hsieh MJ, Yang SF (2020) Impact of the severities of glaucoma on the incidence of subsequent dementia: A population-based cohort study. Int J Environ Res Public Health 17, 2426.

[34] Lee CS, Larson EB, Gibbons LE, Lee AY, McCurry SM, Bowen JD, McCormick WC, Crane PK (2019) Associations between recent and established ophthalmic conditions and risk of Alzheimer's disease. Alzheimers Dement 15, 34-41.

[35] Ou Y, Grossman DS, Lee PP, Sloan FA (2012) Glaucoma, Alzheimer disease and other dementia: A longitudinal analysis. Ophthalmic Epidemiol 19, 285-292.

[36] Su CW, Lin CC, Kao CH, Chen HY (2016) Association between glaucoma and the risk of dementia. Medicine $\mathbf{9 5}$, e2833.

[37] Xiao Z, Wu W, Zhao Q, Liang X, Luo J, Ding D (2020) Association of glaucoma and cataract with incident dementia: A 5-year follow-up in the Shanghai Aging Study. J Alzheimers Dis 76, 529-537.

[38] Keenan TD, Goldacre R, Goldacre MJ (2015) Associations between primary open angle glaucoma, Alzheimer's disease and vascular dementia: Record linkage study. $\mathrm{Br} J$ Ophthalmol 99, 524-527. 
[39] Lin IC, Wang YH, Wang TJ, Wang IJ, Shen YD, Chi NF, Chien LN (2014) Glaucoma, Alzheimer's disease, and Parkinson's disease: An 8-year population-based follow-up study. PLoS One 9, e108938.

[40] Moon JY, Kim HJ, Park YH, Park TK, Park E-C, Kim CY, Lee SH (2018) Association between open-angle glaucoma and the risks of Alzheimer's and Parkinson's diseases in South Korea: A 10-year nationwide cohort study. Sci Rep $\mathbf{8}$, 11161

[41] Tsai DC, Chen SJ, Huang CC, Yuan MK, Leu HB (2015) Age-related macular degeneration and risk of degenerative dementia among the elderly in Taiwan: A population-based cohort study. Ophthalmology 122, 2327-2335 e2322.

[42] Choi S, Jahng WJ, Park SM, Jee D (2020) Association of age-related macular degeneration on Alzheimer or Parkinson disease: A retrospective cohort study. Am J Ophthalmol 210, 41-47.

[43] Lai SW, Lin CL, Liao KF (2014) Cataract may be a nonmemory feature of Alzheimer's disease in older people. Eur J Epidemiol 29, 405-409.

[44] Rodill LG, Exalto LG, Gilsanz P, Biessels GJ, Quesenberry CP, Jr., Whitmer RA (2018) Diabetic retinopathy and dementia in type 1 diabetes. Alzheimer Dis Assoc Disord 32, 125-130.

[45] Exalto LG, Biessels GJ, Karter AJ, Huang ES, Quesenberry CP, Jr., Whitmer RA (2014) Severe diabetic retinal disease and dementia risk in type 2 diabetes. $J$ Alzheimers Dis 42(Suppl 3), S109-117.

[46] Schrijvers EMC, Buitendijk GHS, Ikram MK, Koudstaal PJ, Hofman A, Vingerling JR, Breteler MMB (2012) Retinopathy and risk of dementia: The Rotterdam Study. Neurology 79, 365-370.

[47] Nam GE, Han K, Park SH, Cho KH, Song SJ (2021) Retinal vein occlusion and the risk of dementia: A nationwide cohort study. Am J Ophthalmol 221, 181-189.

[48] Hämäläinen A, Phillips N, Wittich W, Pichora-Fuller MK, Mick P (2019) Sensory-cognitive associations are only weakly mediated or moderated by social factors in the Canadian Longitudinal Study on Aging. Sci Rep 9, 19660.
[49] van der Aa HPA, Comijs HC, Penninx BWJH, van Rens GHMB, van Nispen RMA (2015) Major depressive and anxiety disorders in visually impaired older adults. Invest Ophthalmol Vis Sci 56, 849-854.

[50] Ong SR, Crowston JG, Loprinzi PD, Ramulu PY (2018) Physical activity, visual impairment, and eye disease. Eye 32, 1296-1303.

[51] Carrière I, Delcourt C, Daien V, Pérès K, Féart C, Berr C, Laure Ancelin M, Ritchie K (2013) A prospective study of the bi-directional association between vision loss and depression in the elderly. J Affect Disord 151, 164-170.

[52] Griffiths TD, Lad M, Kumar S, Holmes E, McMurray B, Maguire EA, Billig AJ, Sedley W (2020) How can hearing loss cause dementia? Neuron 108, 401-412.

[53] Mancino R, Martucci A, Cesareo M, Giannini C, Corasaniti MT, Bagetta G, Nucci C (2018) Glaucoma and Alzheimer disease: One age-related neurodegenerative disease of the brain. Curr Neuropharmacol 16, 971-977.

[54] Kaarniranta K, Salminen A, Haapasalo A, Soininen H, Hiltunen M (2011) Age-related macular degeneration (AMD): Alzheimer's disease in the eye? J Alzheimers Dis 24, 615-631.

[55] Jefferis JM, Mosimann UP, Clarke MP (2011) Cataract and cognitive impairment: A review of the literature. $\mathrm{Br} \mathrm{J} \mathrm{Oph}$ thalmol 95, 17-23.

[56] Xue M, Xu W, Ou Y-N, Cao X-P, Tan M-S, Tan L, Yu J-T (2019) Diabetes mellitus and risks of cognitive impairment and dementia: A systematic review and meta-analysis of 144 prospective studies. Ageing Res Rev 55, 100944.

[57] Cheung CY, Chan VTT, Mok VC, Chen C, Wong TY (2019) Potential retinal biomarkers for dementia: What is new? Curr Opin Neurol 32.

[58] Pellegrini M, Bernabei F, Schiavi C, Giannaccare G (2020) Impact of cataract surgery on depression and cognitive function: Systematic review and meta-analysis. Clin Exp Ophthalmol 48, 593-601.

[59] Sperling R, Mormino E, Johnson K (2014) The evolution of preclinical Alzheimer's disease: Implications for prevention trials. Neuron 84, 608-622. 